\title{
Direct numerical simulations of a statistically stationary streamwise periodic boundary layer via the homogenized Navier-Stokes equations
}

\author{
Joseph Ruan $\odot$ and Guillaume Blanquart $\odot$ \\ California Institute of Technology, Pasadena, California 91125, USA
}

\begin{abstract}
(Received 4 February 2020; accepted 6 January 2021; published 5 February 2021)
We demonstrate a method for direct numerical simulations (DNS) of incompressible, flat-plate, zero pressure gradient, turbulent boundary layers, without the use of auxiliary simulations or fringe regions, in a streamwise periodic domain via the homogenized Navier-Stokes equations. This approach is inspired by Spalart's original (1987) method, but improves upon his drawbacks while simplifying the implementation. Most simulations of flat-plate boundary layers require long streamwise domains owing to the slow boundary layer growth and inflow generation techniques. Instead, we use anticipated self-similarity to solve the equations in a normalized coordinate system to allow for streamwise periodicity, similar to Spalart's original method. The resulting integral values, the skin friction coefficient and shape factor, $H_{12}$ and $C_{f}$, are within $\pm 1 \%$ and $\pm 3 \%$ of the empirical fits. The mean profiles show good agreement with spatially developing DNS and experimental results for a wide range of Reynolds numbers from $\operatorname{Re}_{\delta^{*}}=1460$ to 5650. The method manages to reduce computational costs by an estimated one to two orders of magnitude.
\end{abstract}

DOI: 10.1103/PhysRevFluids.6.024602

\section{INTRODUCTION}

Turbulent boundary layers have played a crucial role in numerous engineering applications since Prandtl introduced the concept of the boundary layer in 1904 [1]. Wall-bounded flows contain a wealth of open questions from the near-wall generation cycle [2] to the large scale structures [3] and even the precise nature of the logarithmic layer [4]. All of these phenomena more fully manifest themselves in higher Reynolds number flows. To thoroughly investigate them from a computational perspective, there is an ever-present need for simulations to match or exceed experimental Reynolds numbers.

At the moment, pipe and channel flow direct numerical simulations (DNS) have been able to do so. Lee and Moser (Ref. [4]) conducted a channel DNS at a friction Reynolds number of 5186, and, for comparison, experimental channel measurements by Schultz and Flack (Ref. [5]) were at a friction Reynolds number of 6000. In contrast, boundary layer DNS have only been able to reach Reynolds numbers of at most $\operatorname{Re}_{\delta^{*}}=13000$ [6], while modern boundary layer experiments regularly achieve far higher Reynolds numbers, up to $\operatorname{Re}_{\delta^{*}} \approx 120000$ [7]. We define the Reynolds number for boundary layers by $\operatorname{Re}_{\delta^{*}}=u_{\infty} \delta^{*} / \nu$, with the displacement thickness $\delta^{*}$, the free-stream streamwise velocity $u_{\infty}$, and the kinematic viscosity $v$. As an example, to verify numerically the Kármán constant of the logarithmic region, one would need a boundary layer DNS with a Reynolds number at least one order of magnitude larger than the current state-of-the-art [8]. This status quo has two origins: the need for inflow boundary conditions and the growing nature of the boundary layer. Previous computational solutions can be classified into two main categories based on their treatments of both issues.

Method No. 1: Inflow generation. Recognizing that simulation of transition is computationally expensive and complex [9], and long streamwise domains are required to reach high Reynolds 
TABLE I. Summary of computational characteristics of previously published numerical approaches.

\begin{tabular}{lccc}
\hline \hline Method & $\begin{array}{c}\text { Statistically } \\
\text { homogeneous (in } x)\end{array}$ & $\begin{array}{c}\text { Statistically } \\
\text { stationary }\end{array}$ & $\begin{array}{c}\text { Closed } \\
\text { form }\end{array}$ \\
\hline Inflow generation & - & $\mathrm{x}$ & - \\
Temporal DNS & $\mathrm{x}$ & - & $\mathrm{x}$ \\
Spalart (1988) & $\mathrm{x}$ & $\mathrm{x}$ & - \\
Proposed method & $\mathrm{x}$ & $\mathrm{x}$ & $\mathrm{x}$ \\
\hline \hline
\end{tabular}

numbers, multiple studies have used inflow generation techniques to bypass transition and initialize their simulations with much higher Reynolds number inflows. There have been several popular methods such as synthetic generation methods [10], strong recycling methods [11], and weak recycling methods [12-16]. While these methods have been used to great effect, they still suffer from multiple computational challenges.

First, current state-of-the-art methods still waste large portions of the computational domain as a result of inflow generation techniques. The recycling domain alone can take up to $25 \%$ of the domain [12]. Synthetic methods can overcome these recycling costs, but Sillero et al. [16] showed that all inflow generation methods have an additional "eddy-turnover recovery length" over which the simulation is heavily influenced by the inflow generation method. The authors found that for over $25 \%$ of their so-called "production" domain, none of the simulation statistics match empirical results. Overall, these inflow-generation methods underexploit available computational resources.

Second, as the boundary layer grows in space $\left(d \delta^{*} / d x>0\right)$, momentum is displaced away from the wall, and mass leaves through the top surface of the computational domain $\left(V_{\infty}>0\right)$. To enforce global mass conservation in incompressible DNS, an a priori streamwise dependence of $V_{\infty}$ must be imposed over the entire domain [12,15,16]. Unfortunately, the imposition of $V_{\infty}$ enforces a particular boundary layer growth rate. This is easily seen by integrating continuity $\left(V_{\infty}=U_{\infty} \frac{d \delta^{*}}{d x}\right)$. Under these conditions, one might ask if boundary layer simulations with fixed growth rates are true DNS.

Method No. 2: Streamwise periodicity. One of the crucial differences between channel and boundary layer flows from a numerical point of view is the streamwise growth of the boundary layer. A statistically stationary, periodic method for boundary layer simulations would be ideal since it would bypass the use of an inflow generation technique. This was proposed by Spalart [17] who used a multiscale decomposition of velocity and subsequently scaled the wall-normal coordinate to take advantage of the flow's self-similarity. In the new coordinates, the mean quantities were only functions of the rescaled wall-normal coordinate, and so Spalart obtained a streamwise periodic and statistically stationary boundary layer. Unfortunately, this transformation generated several additional terms in the governing equations that could not be closed without performing auxiliary simulations of boundary layer flow for lower Reynolds numbers.

Eschewing these unclosed source terms, several studies [18-21] have nevertheless sought to impose streamwise periodic boundary conditions, attempting to leverage the boundary layer's "quasiparallel" nature. While the numerical solution is now homogeneous in the streamwise direction (owing to the periodicity), it can never reach a statistically stationary state. These "temporal" DNS (as opposed to the previously discussed "spatial" DNS) have a limited sample time $\left(<1 \delta_{99} / u_{\tau}\right)$ before the boundary layer thickness drifts significantly.

In summary, there is currently no immediate computational framework for the simulation of boundary layers that is (1) statistically homogeneous in the streamwise direction, (2) statistically stationary, and (3) fully closed (see Table I). The objective of the present work is to propose a framework to improve on Spalart's preliminary method such that it does not rely on auxiliary simulations for closure. The overall concept remains the same: to use periodic boundary conditions and a scaling enforced by a coordinate transformation to keep the boundary layer statistically stationary. 
We will detail the principles of the transformation in Sec. II and conduct extended domain simulations in Sec. III to justify streamwise statistical homogeneity. We highlight the numerical methods in Sec. IV. In Sec. V, we present validation and comparisons to DNS data from Refs. [16,22] and empirical fits by Refs. [23,24]. Finally, in Sec. VI, we discuss computational savings.

\section{ANALYSIS OF STATIONARY BOUNDARY LAYER}

The goal of this section is to describe the proposed method of simulating flat-plate turbulent boundary layers. It begins with a description of the spatial transformation, and a discussion of the simplifications leading to the final set of equations.

\section{A. Transformation of the Navier-Stokes equations}

The flat-plate turbulent boundary layer is analyzed in the Cartesian coordinate system using index notation such that the velocity components in the Cartesian streamwise $\left(x_{1}\right)$, wall-normal $\left(x_{2}\right)$, and spanwise $\left(x_{3}\right)$ directions are $u_{1}, u_{2}$, and $u_{3}$, respectively. With pressure and density as $P$ and $\rho$, respectively, the incompressible Navier-Stokes equations for mass and momentum conservation are

$$
\begin{gathered}
\frac{\partial u_{j}}{\partial x_{j}}=0, \\
\frac{\partial u_{i}}{\partial t}+u_{j} \frac{\partial u_{i}}{\partial x_{j}}=-\frac{1}{\rho} \frac{\partial P}{\partial x_{i}}+v \frac{\partial^{2} u_{i}}{\partial x_{j}^{2}} .
\end{gathered}
$$

We now apply a coordinate transformation from $x_{i}$ to $\xi_{i}$ which rescales the wall-normal coordinate by a streamwise varying $C^{2}$ function $q=q\left(x_{1}\right)$.

$$
\xi_{1}=x_{1}, \quad \xi_{2}=\frac{q_{0}}{q} x_{2}, \quad \xi_{3}=x_{3},
$$

where $q_{0}=q\left(x_{0}\right)$ is a normalization constant that is yet to be determined. Applying this coordinate transformation directly to the Navier-Stokes equations yields the following set of equations for mass and momentum conservation.

$$
\begin{gathered}
\frac{\partial u_{j}}{\partial \xi_{j}}=\xi_{2} \frac{q^{\prime}}{q} \frac{\partial u_{1}}{\partial \xi_{2}}+H_{c}, \\
\frac{\partial u_{i}}{\partial t}=-u_{j} \frac{\partial u_{i}}{\partial \xi_{j}}-\frac{1}{\rho} \frac{\partial P}{\partial \xi_{i}}+v \frac{\partial^{2} u_{i}}{\partial \xi_{j}^{2}}+\xi_{2} \frac{q^{\prime}}{q} u_{1} \frac{\partial u_{i}}{\partial \xi_{2}}+H_{p}\left(u_{i}\right)+H_{\nu}\left(u_{i}\right),
\end{gathered}
$$

where

$$
\begin{gathered}
H_{c}=\left(1-\frac{q_{0}}{q}\right) \frac{\partial u_{2}}{\partial \xi_{2}} \\
H_{p}\left(u_{i}\right)=\delta_{1 i} \frac{1}{\rho} \frac{q^{\prime}}{q} \xi_{2} \frac{\partial P}{\partial \xi_{2}}+\left(\frac{q-q_{0}}{q}\right)\left(\delta_{2 i} \frac{1}{\rho} \frac{\partial P}{\partial \xi_{2}}+u_{2} \frac{\partial u_{i}}{\partial \xi_{2}}\right), \\
H_{v}\left(u_{i}\right)=v\left[1-\left(\frac{q_{0}}{q}\right)^{2}+\left(\xi_{2} \frac{q^{\prime}}{q}\right)^{2}\right] \frac{\partial^{2} u_{i}}{\partial \xi_{2}^{2}} \\
+v\left[2\left(\frac{q^{\prime}}{q}\right)^{2}-\frac{q^{\prime \prime}}{q}\right] \xi_{2} \frac{\partial u_{i}}{\partial \xi_{2}}-2 v \xi_{2} \frac{q^{\prime}}{q} \frac{\partial^{2} u_{i}}{\partial \xi_{1} \partial \xi_{2}},
\end{gathered}
$$

where $\delta_{i j}$ is the delta-Dirac function, $H_{c}$ is an additional continuity term, $H_{p}$ contains convective and pressure additional metric terms, and $H_{v}$ contains the viscous metric terms. The equations as shown 


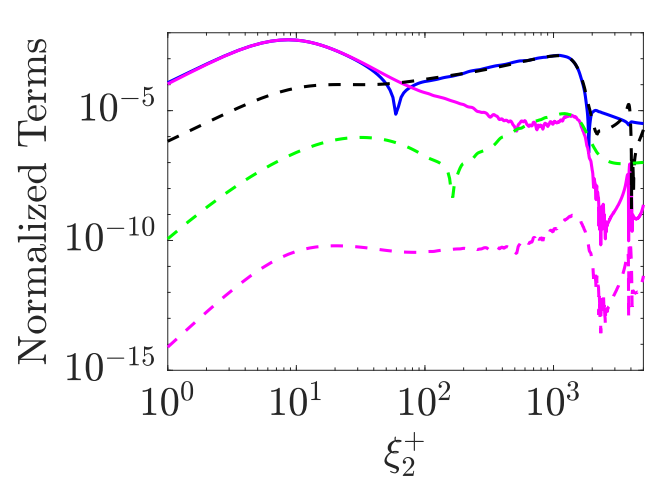

(a) streamwise momentum budget

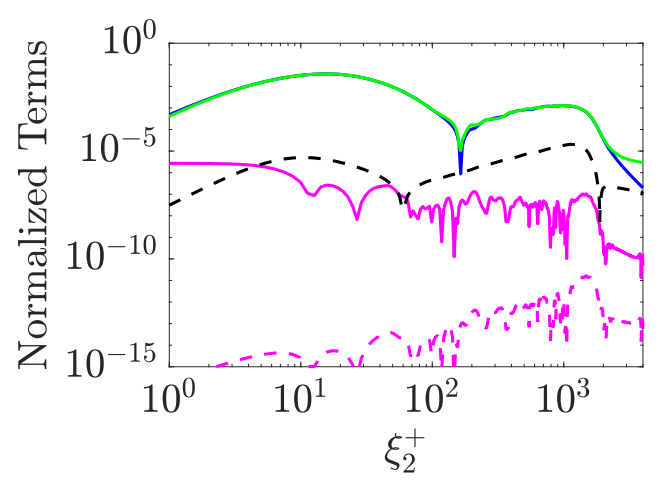

(b) wall-normal momentum budget

FIG. 1. Budgets of (a) streamwise momentum and (b) wall-normal momentum equations from DNS data (Ref. [16]). Lines: (solid blue) $\left|\left\langle C_{\mathrm{NS}}\right\rangle_{\xi_{3}, t}\right|$; (solid magenta) $\left|\left\langle V_{\mathrm{NS}}\right\rangle_{\xi_{3}, t}\right|$; (solid green) $\left\langle P_{\mathrm{NS}}\right\rangle_{\xi_{3}, t}$; (dashed black) $\mid\langle\text { Src }\rangle_{\xi_{3}, t} \mid$; (dashed green) $\left|\left\langle H_{p}\right\rangle_{\xi_{3}, t}\right|$; (dashed magenta) $\left|\left\langle H_{\nu}\right\rangle_{\xi_{3}, t}\right|$.

are exact and equivalent to the original Navier-Stokes equations. At the moment, $q\left(x_{1}\right)$ still requires a closure equation for the transformed Navier-Stokes equations to be complete. There are several possible choices to choose from such as the $99 \%$ boundary layer thickness $\delta_{99}$, the displacement thickness $\delta^{*}$, and the momentum thickness $\theta$. Each choice yields a unique and mathematically valid coordinate transformation.

\section{B. a priori analysis}

We perform a budget analysis of the streamwise and wall-normal momentum equations (4)-(7) and the turbulent kinetic energy equations $(\mathrm{C} 1)-(\mathrm{C} 4)$. This a priori analysis is performed using the DNS data from Ref. [16] near $\operatorname{Re}_{\theta_{0}}=4000$.

Any a priori analysis of Eqs. (4)-(7) requires estimates for the function $q\left(x_{1}\right)$. This function is here approximated by $\theta\left(x_{1}\right)$, and justification for the estimate will be given in Sec. II C. Empirical fits from [23] provide value for $\frac{\theta^{\prime}}{\theta} \theta_{0}$ at $\operatorname{Re}_{\theta_{0}}=4000$.

We start with the streamwise momentum equation. First, we evaluate all terms at $q=q_{0}$. This reduces $H_{p}$ to a single term, removes a term from $H_{\nu}$, and completely eliminates $H_{c}$. We also group the main convective terms, $C_{N S}=u_{1} \partial u_{1} / \partial \xi_{1}+u_{2} \partial u_{1} / \partial \xi_{2}+u_{3} \partial u_{1} / \partial \xi_{3}$, and the main viscous terms, $V_{N S}=v\left(\partial^{2} u_{1} / \partial \xi_{1}^{2}+\partial^{2} u_{1} / \partial \xi_{2}^{2}+\partial^{2} u_{1} / \partial \xi_{3}^{2}\right)$. The source term for this equation is given by $\mathrm{Src}=q^{\prime} / q \xi_{2} u_{1} \partial u_{1} / \partial \xi_{2}$. Figure 1(a) shows the budget analysis of the streamwise momentum equation. All terms have been first averaged over time and spanwise coordinate, represented by $\langle\cdot\rangle_{\xi_{3}, t}$, and then the inner-scaled absolute values of these averages are plotted. Notably, the main convective terms change sign near the wall to balance the main viscous terms.

As expected, the most dominant terms are the convective and viscous terms, $C_{\mathrm{NS}}$ and $V_{\mathrm{NS}}$. Furthermore, Fig. 1(a) clearly shows that the convective metric term $\xi_{2} u_{1}\left(q^{\prime} / q\right) \partial u_{1} / \partial \xi_{2}$ is the most dominant of the additional metric terms. It balances the main convective terms near the end of the logarithmic region and throughout the wake region $\left(80<\xi_{2}^{+}<2000\right)$. In contrast, the viscous metric term $H_{v}$ is over six orders of magnitude smaller than the streamwise convective term throughout the entire boundary layer. Similarly, the $H_{p}$ term is at least three orders of magnitude smaller than the streamwise convective metric term until the very end of the wake region near the free stream. From an a priori perspective, the neglecting of $H_{v}$ and $H_{p}$ is justified.

One can also apply a similar analysis to the wall-normal momentum equation. In this case, the convective terms are bundled as $C_{N S}=u_{1} \partial u_{2} / \partial \xi_{1}+u_{2} \partial u_{2} / \partial \xi_{2}+u_{3} \partial u_{2} / \partial \xi_{3}$, and the main 


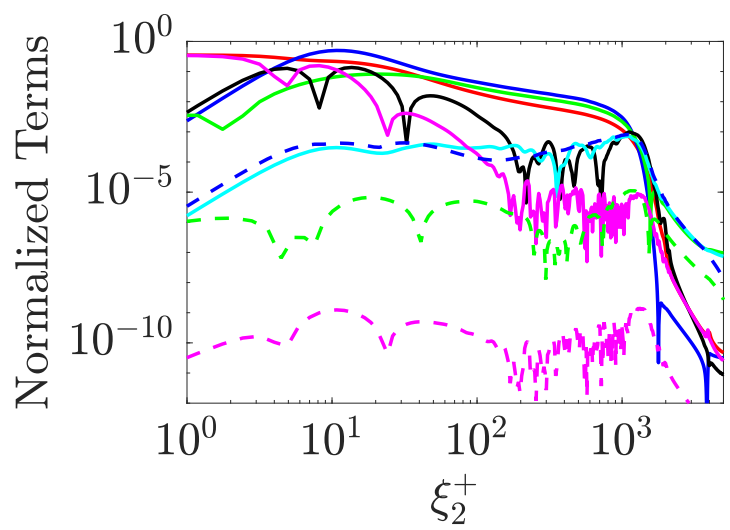

FIG. 2. Mean turbulent kinetic energy budget from DNS data (Ref. [16]). Lines: (solid blue) turbulent production; (red) dissipation; (green) pressure diffusion; (black) turbulent diffusion; (cyan) advection; (magenta) viscous diffusion; (dashed green) $H_{p}$ contribution; (dashed magenta) $H_{v}$ contribution; (dashed blue) metric source term contribution.

viscous terms are collected in $V_{\mathrm{NS}}=v\left(\partial^{2} u_{2} / \partial \xi_{1}^{2}+\partial^{2} u_{2} / \partial \xi_{2}^{2}+\partial^{2} u_{2} / \partial \xi_{3}^{2}\right)$. The remaining terms are the mean pressure gradient term $P_{\mathrm{NS}}=1 / \rho \partial P / \partial \xi_{2}$ and the source term $\mathrm{Src}=q^{\prime} / q \xi_{2} u_{1} \partial u_{2} / \partial \xi_{2}$. Figure 1(b) shows the budget analysis of the wall-normal momentum equation. Again, all terms have been first averaged over time and spanwise coordinate, and then the inner-scaled absolute values of these averages are plotted.

In this case, the balance between the pressure and the convective terms dominates the entire budget. The magnitude of the source term is between that of the convective and viscous terms. The viscous metric term remains seven orders of magnitude smaller than the pressure and convective terms throughout the boundary layer and thus can justifiably be neglected in the wall-normal momentum equation. Near the free stream $\left(\xi_{2}^{+}>2000\right)$, the source term and the convective term balance the pressure gradient term.

Finally, one can apply a similar analysis to the turbulent kinetic energy equation and track relative contributions of the $H_{p}, H_{\nu}$, and the source term. The results are shown in Fig. 2. The metric source term contribution balances the turbulent advection term which is at least three orders of magnitude below the dominant budget terms. Throughout the boundary layer, the contributions of both $H_{v}$ and $H_{p}$ to the kinetic energy budget remain several orders of magnitude lower than the dominant budget terms. From an a priori perspective, their impact on turbulent intensities is negligible.

\section{Simplified equations and closure}

We can now make the following two critical assumptions:

(1) The governing equations evaluated at $q\left(x_{1}\right)=q_{0}$ are valid for a narrow streamwise domain centered at $x_{1}=x_{0}$.

(2) There exists a function $q\left(x_{1}\right)$ such that ensemble-averaged quantities are both statistically stationary and statistically homogeneous in the $\xi_{1}, \xi_{3}$ directions. to

Given both assumptions and with the neglecting of $H_{v}$ and $H_{p}$, the governing equations simplify

$$
\begin{gathered}
\frac{\partial u_{j}}{\partial \xi_{j}}=\xi_{2} \frac{q_{0}^{\prime}}{q_{0}} \frac{\partial u_{1}}{\partial \xi_{2}}, \\
\frac{\partial u_{i}}{\partial t}=-u_{j} \frac{\partial u_{i}}{\partial \xi_{j}}-\frac{1}{\rho} \frac{\partial P}{\partial \xi_{i}}+v \frac{\partial^{2} u_{i}}{\partial \xi_{j}^{2}}+\xi_{2} \frac{q_{0}^{\prime}}{q_{0}} u_{1} \frac{\partial u_{i}}{\partial \xi_{2}} .
\end{gathered}
$$


These are the final governing equations to be solved via streamwise periodic simulation. Section III presents an a posteriori analysis justifying both the neglecting of $H_{v}$ and $H_{p}$ and the streamwise statistical homogeneity of Eqs. (9) and (10).

The use of both assumptions and the neglecting of $H_{v}$ and $H_{p}$ mean that the governing equations can be more accurately described as homogenized Navier-Stokes equations (HNSE). Consequently, simulations utilizing this set of equations are still DNS but do not directly solve the NSE. The rest of the document seeks to compare the solutions of the HNSE to experimental and numerical solutions to the NSE.

We now seek to generate a closure equation for $q_{0}^{\prime} / q_{0}$ by considering the $\xi_{2}$ integrated continuity and streamwise momentum equations in conservative form.

$$
\begin{gathered}
\int_{0}^{\infty}\left(\frac{\partial u_{1}}{\partial \xi_{1}}+\frac{\partial u_{3}}{\partial \xi_{3}}\right) d \xi_{2}+u_{2, \infty}=\frac{q_{0}^{\prime}}{q_{0}} \int_{0}^{\infty}\left(\xi_{2} \frac{\partial u_{1}}{\partial \xi_{1}}\right) d \xi_{2} \\
\int_{0}^{\infty}\left(\frac{\partial \rho u_{1}}{\partial t}+\frac{\partial \rho u_{1} u_{j}}{\partial \xi_{j}}+\frac{\partial P}{\partial \xi_{1}}\right) d \xi_{2} \\
=\frac{q_{0}^{\prime}}{q_{0}} \int_{0}^{\infty}\left(\xi_{2} \frac{\partial \rho u_{1} u_{1}}{\partial \xi_{1}}\right) d \xi_{2}+\int_{0}^{\infty} \mu \frac{\partial^{2} u_{1}}{\partial \xi_{k}^{2}} d \xi_{2} .
\end{gathered}
$$

We now ensemble average Eqs. (11) and (12) and denote ensemble-averaged quantities by $\langle\cdot\rangle$. Applying assumption 2 yields the following equations.

$$
\begin{aligned}
& u_{2, \infty}=\frac{q_{0}^{\prime}}{q_{0}} \int_{0}^{\infty}\left(u_{1, \infty}-\left\langle u_{1}\right\rangle\right) d \xi_{2}=\frac{q_{0}^{\prime}}{q_{0}} u_{1, \infty} \delta^{*}, \\
& u_{1, \infty} u_{2, \infty}+\frac{\tau_{w}}{\rho}=\frac{q_{0}^{\prime}}{q_{0}} \int_{0}^{\infty}\left(u_{1, \infty}{ }^{2}-\left\langle u_{1} u_{1}\right\rangle\right) d \xi_{2},
\end{aligned}
$$

where $\tau_{w}=\left.\mu\left\langle\partial u_{1} / \partial \xi_{2}\right\rangle\right|_{\xi_{2}=0}$ is the wall shear stress. Some further manipulation gives our final closure equation.

$$
\frac{q_{0}^{\prime}}{q_{0}}=\frac{\tau_{w} / \rho}{\int_{0}^{\infty}\left(u_{1, \infty}\left\langle u_{1}\right\rangle-\left\langle u_{1} u_{1}\right\rangle\right) d \xi_{2}} .
$$

This expression fully closes the simplified governing equations [Eqs. (9) and (10)] and is used in the streamwise periodic numerical simulations of Sec. IV.

Before discussing the results, it is interesting to estimate a priori the right-hand side of the above equation. The von Kármán momentum integral equation for a flat-plate boundary layer estimates the growth rate of the momentum thickness by

$$
\theta^{\prime}=\frac{\tau_{w}}{\rho u_{1, \infty}^{2}} .
$$

By definition,

$$
u_{1, \infty}^{2} \theta=\int_{0}^{\infty}\left(u_{1, \infty}\left\langle u_{1}\right\rangle-\left\langle u_{1}\right\rangle\left\langle u_{1}\right\rangle\right) d \xi_{2} .
$$

Assuming that $\left\langle u_{1}\right\rangle^{2} \approx\left\langle u_{1}^{2}\right\rangle$, the von Kármán momentum integral equation becomes

$$
\frac{\theta^{\prime}}{\theta} \approx \frac{\tau_{w} / \rho}{\int_{0}^{\infty}\left(u_{1, \infty}\left\langle u_{1}\right\rangle-\left\langle u_{1} u_{1}\right\rangle\right) d \xi_{2}}=\frac{q_{0}^{\prime}}{q_{0}} .
$$


TABLE II. DNS parameters for the streamwise nonperiodic turbulent boundary layer simulation cases.

\begin{tabular}{lcccc}
\hline \hline Dataset & $L_{x}$ & Governing equations & Closure equation & Sample time $\delta_{99} / u_{\tau}$ \\
\hline BL_Cart & $15 \delta_{99}$ & Eqs. (1) and (2) & None & 15 \\
BL_Full & $15 \delta_{99}$ & Eqs. (4)-(8) & Empirical value & 30 \\
BL_Simp & $15 \delta_{99}$ & Eqs. (9) and (10) & Empirical value & 30 \\
BL_Plus & $15 \delta_{99}$ & Eqs. (4)-(8) & $1.1 \times$ empirical value & 15 \\
BL_Minus & $15 \delta_{99}$ & Eqs. (9) and (10) & $0.9 \times$ empirical value & 15 \\
BL_Per & $7.5 \delta_{99}$ & Eqs. (9) and (10) & Eq. (15) & 30 \\
\hline \hline
\end{tabular}

This completes the intuition that $q(x)$ scales like $\theta(x)$ and furthermore provides an estimate for $q_{0}^{\prime} / q_{0}$.

$$
\frac{q_{0}^{\prime}}{q_{0}} \approx \frac{\theta^{\prime}}{\theta}=\frac{1}{2 \delta^{*}} C_{f} H_{12}
$$

where $C_{f}=\tau_{w} /\left(\frac{1}{2} \rho u_{1, \infty}^{2}\right)$ is the skin-friction coefficient and $H_{12}=\delta^{*} / \theta$ is the shape factor.

Note that the closure equation ensures a statistically stationary flow and consequently the solution will be specific to a single Reynolds number. This is in direct contrast with recycling and rescaling methods which solve for a range of Reynolds numbers but also use flow at high Reynolds number stations as a substitute for a low Reynolds number inflow. The net effect of the closure equation [Eq. (15)] is to allow the current method to avoid unphysical inflows by focusing on a single Reynolds number.

\section{SPATIALLY DEVELOPING SIMULATIONS}

We conduct six sets of boundary layer simulations, each solving a different set of governing equations and boundary conditions, summarized in Table II. The results are used to justify assumptions (1) and (2), and the simplifications made to the governing equations in Sec. II B.

\section{A. Simulations and numerical methods}

With the exception of BL_Per, all of the cases have streamwise nonperiodic boundaries in an inflow/outflow setup. Case BL_Per corresponds to the most "modified" case: it solves Eqs. (9) and (10) with Eq. (15) and implements streamwise periodic boundary conditions. Case BL_Simp also solves Eqs. (9) and (10) but does not use streamwise periodic boundary conditions. Case BL_Full solves Eqs. (4)-(8) and contains all of the previously neglected terms. Cases BL_Full and BL_Simp use empirical relations for low Reynolds number [24] for the closure of $q_{0}^{\prime} / q_{0}$ by approximating $q \approx \theta$. Cases BL_Plus and BL_Minus differ from BL_Simp by using a closure for $q_{0}$ artificially increased and decreased by 10\%, respectively. Finally, case BL_Cart solves the regular Cartesian Navier-Stokes equations [Eqs. (1) and (2)].

All of the cases have periodic spanwise directions and nonperiodic wall-normal directions. The bottom of the domain is treated with a no-slip boundary condition, and the top of the computational domain is treated with a Neumann boundary condition. Each of the five inflow/outflow cases use planes from case BL_Per as an inflow (at $\xi_{1}=0$ ). All of the streamwise nonperiodic cases use convective outflow conditions at the streamwise outlet and have mass conservation conducted at the streamwise outlet. In contrast, case BL_Per has mass conservation conducted at the wall-normal outlet. The top of the computational domain requires vertical transpiration for all six cases. BL_Cart imposes a transpiration velocity given by Ref. [23], similar to Ref. [16]. For the remaining cases, Eq. (11) shows that any closure for $q_{0}^{\prime} / q_{0}$ directly provides a value for $u_{2, \infty}$.

All of the cases have the same spanwise length of $L_{z}=2.6 \delta_{99}$ and wall-normal height of $L_{y}=3.4 \delta_{99}$. They all have the same spatial resolution: $\Delta x^{+}=9, \Delta y_{\min }^{+}=0.3, \Delta z^{+}=6$. The key 


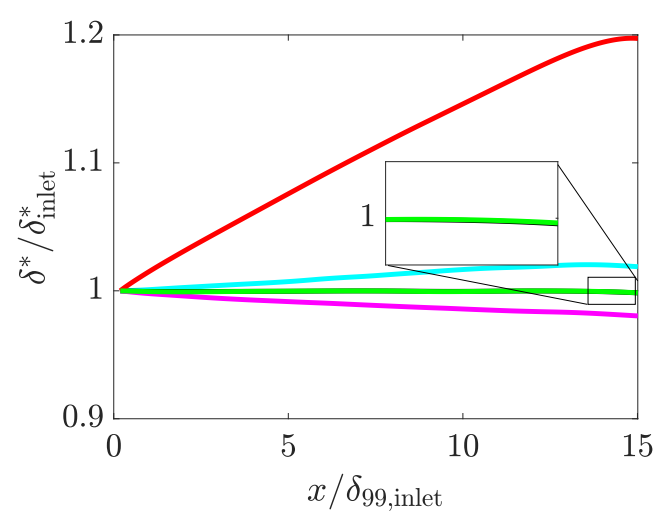

(a) Displacement thickness

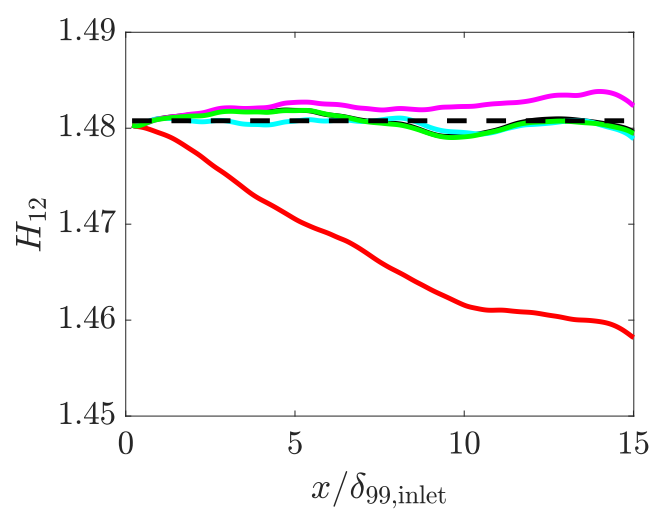

(c) Shape factor

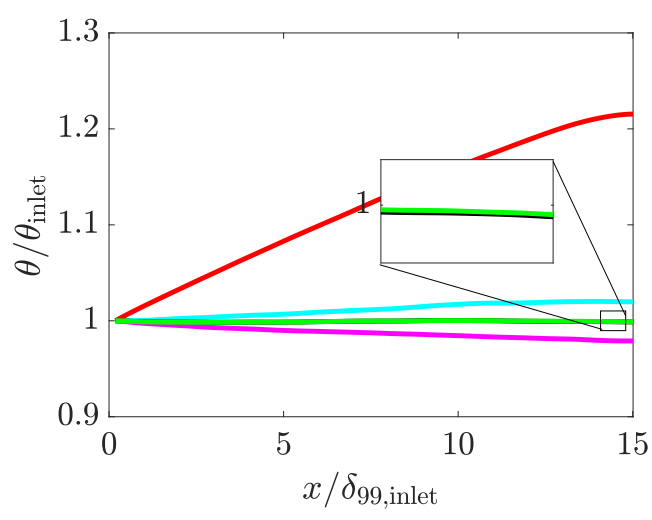

(b) Momentum thickness

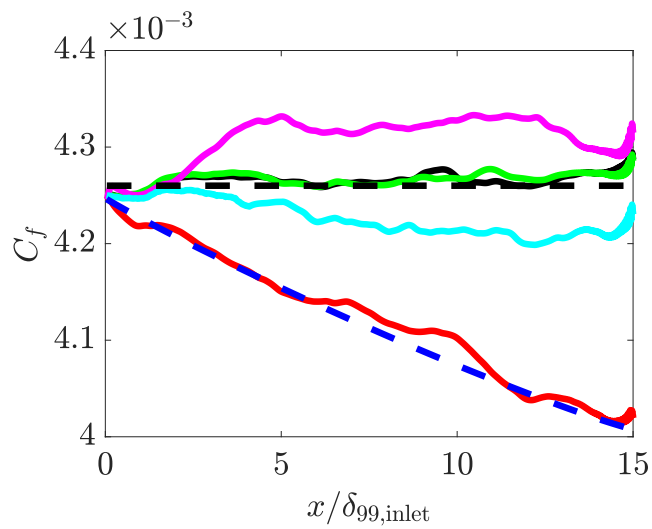

(d) Skin friction coefficient

FIG. 3. Streamwise variation of (a) displacement thickness $\left(\delta^{*}\right)$, (b) momentum thickness $(\theta)$, (c) shape factor $\left(H_{12}\right)$, and (d) skin friction coefficient $\left(C_{f}\right)$. Lines: (solid black) BL_Full; (solid green) BL_Simp; (solid cyan) BL_Minus; (solid magenta) BL_Plus; (solid red) BL_Cart; (dashed black) BL_Per; (dashed blue) scaled empirical fit [24] to match inlet skin friction coefficient.

difference between the streamwise periodic and streamwise nonperiodic cases is the streamwise domain length. BL_Per has a domain length of $L_{x}=7.5 \delta_{99}$, whereas the rest of the cases have a domain length of $L_{x}=15 \delta_{99}$. It is known from [25] that the flow recovers from this particular inflow technique after $\sim 4-5 \delta_{99}$. Accounting for potential outflow effects of at most $\sim 2 \delta_{99}$, this leaves about $8 \delta_{99}$ of uncontaminated statistics.

Each set of governing equations is solved using the computational solver NGA [26]. The numerical code solves the conservative-variable formulation of the low-Mach Navier-Stokes equations with staggered finite difference operators and uses a fractional step method to enforce continuity. The code is run fully second order in space and time.

\section{B. Results}

Figures 3(a) and 3(b) present the normalized displacement thickness and momentum thickness averaged in $\xi_{3}$ and in time for the streamwise nonperiodic cases. Naturally, each of the streamwise nonperiodic simulation cases is affected by the convective outflow condition, most clearly seen in case BL_Cart. Each of the displacement thickness and momentum thickness plots deviate in slope at about $1 \delta_{99}$ from the streamwise outlet. Because BL_Cart represents a spatially developing boundary layer, the displacement thickness increases from its original inflow value. The present increase by 
about $20 \%$ is expected given that $d \delta^{*} / d x=u_{2, \infty} / u_{1, \infty}$ and the imposed value of $u_{2, \infty} / u_{1, \infty} \approx 3 \times$ $10^{-3}$. Cases BL_Full and BL_Simp are indistinguishable and show relatively constant values of $\delta^{*}$ and $\theta$ with fluctuation magnitudes of $\pm 0.3 \%$ of the inflow nominal value. The thicknesses of BL_Plus and BL_Minus show immediate departures from the nominal value, by approximately $2 \%$ of the original inflow value.

References $[9,27]$ underscore the need for consistency when comparing DNS profiles of boundary layers. For the sake of comparison, integral and global quantities are computed as described by Ref. [9]. Specifically, the shape factor, $H_{12}$, is evaluated as

$$
H_{12}=\frac{\int_{0}^{\delta_{99}}\left(1-\bar{u}_{1} / u_{1, \infty}\right) d \xi_{2}}{\int_{0}^{\delta_{99}}\left(\bar{u}_{1} / u_{1, \infty}\right)\left(1-\bar{u}_{1} / u_{1, \infty}\right) d \xi_{2}},
$$

where, for the remainder of this section, the overbar represents temporal and spanwise averaging. Similarly, the wall shear stress is evaluated by $\tau_{w}=\mu \partial \overline{u_{1}} /\left.\partial \xi_{2}\right|_{\xi_{2}=0}$. Figure 3(c) presents the shape factor for the nonstreamwise periodic cases. BL_Cart has a shape factor that monotonically drops by $2 \%$ from its inflow value, as expected from empirical fits by [23] with respect to Reynolds number. BL_Plus and BL_Minus also exhibit a slowly varying shape factor, changing by approximately $\pm 0.3 \%$ from the inflow value. This is in contrast with BL_Full and BL_Simp, whose shape factors are virtually identical and do not exhibit major mean variations.

Figure 3(d) presents the skin friction coefficient, averaged in $\xi_{3}$ and in time for the nonstreamwise periodic cases. Case BL_Cart features a decreasing skin friction coefficient over the domain, consistent with increasing Reynolds number. The skin friction coefficients of BL_Plus, BL_Minus, BL_Full, and BL_Simp have fluctuations of about $1 \%$ of their expected mean value. Any variations of the value with streamwise distance are masked by these fluctuations. Again, BL_Simp and BL_Full are virtually indistinguishable.

Figure 4 shows temporal and spanwise-averaged profiles of $\overline{u_{1}}, u_{1, \mathrm{rms}}, u_{2, \mathrm{rms}}$, and $-\overline{u_{1}^{\prime} u_{2}^{\prime}}$ from case BL_Simp and BL_Full. These profiles are extracted from three streamwise locations: near the inlet $\left(\xi_{1}=0\right)$ and outlet $\left(\xi_{1}=13 \delta_{99}\right)$ for case BL_Simp, and in the middle of the domain $\left(\xi_{1}=7.5 \delta_{99}\right)$ for both cases BL_Simp and BL_Full. The mean streamwise velocity profiles are within $\pm 0.5 \%$ of each other. The streamwise and wall-normal rms collapse within $\pm 1 \%$ of each other. The Reynolds stress profiles show a strong collapse in both the inner and outer regions.

Overall, these streamwise nonperiodic simulations show that under a rescaling by $q(x)$, the resulting flow does not feature immediately observable streamwise inhomogeneities over a sizable streamwise domain. The neglecting of $H_{\nu}$ and $H_{p}$ terms and the use of streamwise periodic conditions under Eqs. (9) and (10) are consequently well justified.

\section{NUMERICAL SETUP OF STREAMWISE PERIODIC SIMULATIONS}

The present section outlines the simulations conducted in streamwise periodic domains. It clarifies domain constraints and initial conditions, and describes additional numerical techniques used during simulation.

\section{A. Simulation cases}

We now solve Eqs. (9) and (10) with streamwise periodic boundary conditions for four different Reynolds numbers, summarized in Table III. Case BL1460 is equivalent to BL_Per. Cases BL2830, BL3550, and BL5650 were chosen for direct comparison against the DNS and experiments of [22].

The domain size, $\left(L_{x}, L_{y}, L_{z}\right)$, is determined primarily by the sizes of the largest turbulent structures. The pressure fluctuations are known to reach the furthest out of the boundary layer to about $2.4 \delta_{99}$ [28], setting the minimum requirement for wall-normal height. We set our domain height to $18 \delta^{*} \sim 3 \delta_{99}$ to fully capture these fluctuations. Since low-momentum streaks are approximately $0.5 \delta_{99}$ in width [15,29], we opt for a spanwise width of $14 \delta^{*} \sim 2.5 \delta_{99}$, which is comparable to the domain size of Ref. [22]. The large-scale motions corresponding to bulges or hairpin packets have a maximum streamwise length of $3 \delta_{99}$ [30-34]. In contrast, the very large-scale motions have lengths 


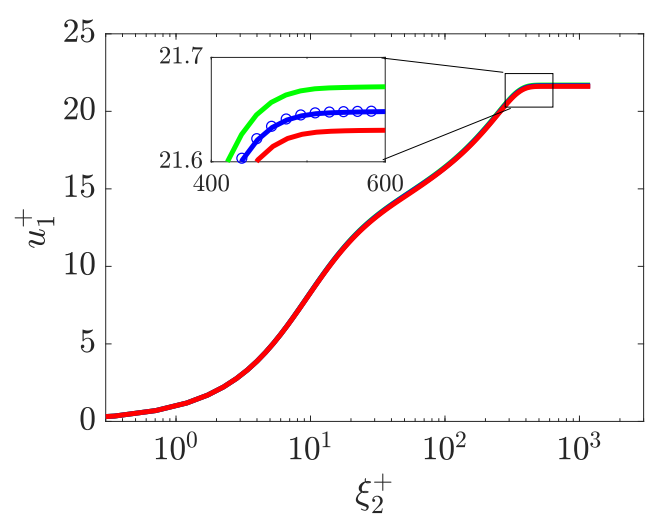

(a) streamwise velocity

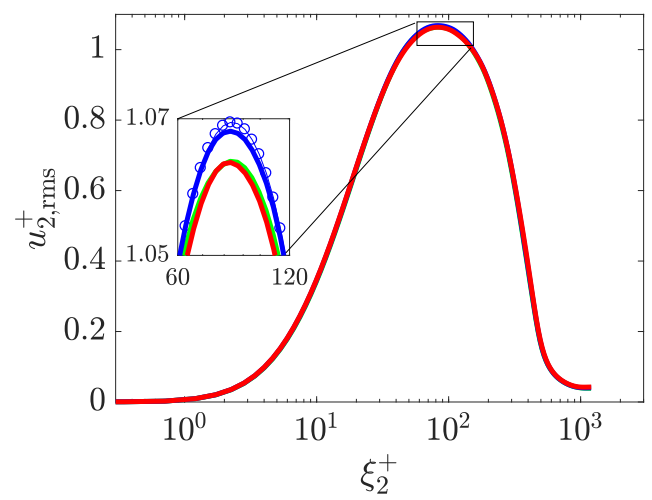

(c) Wall-normal rms

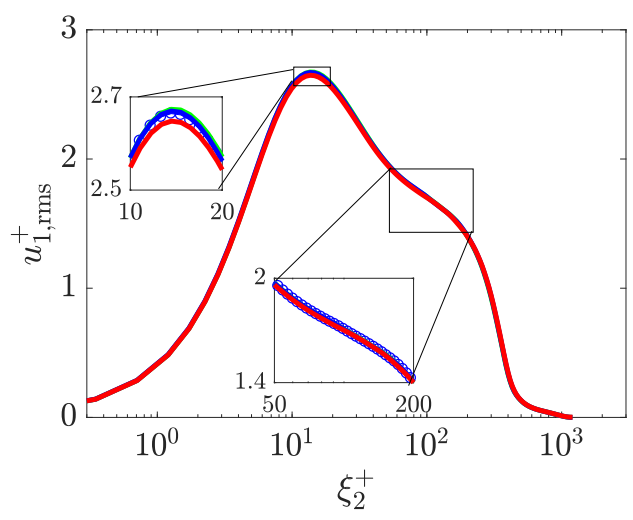

(b) streamwise rms

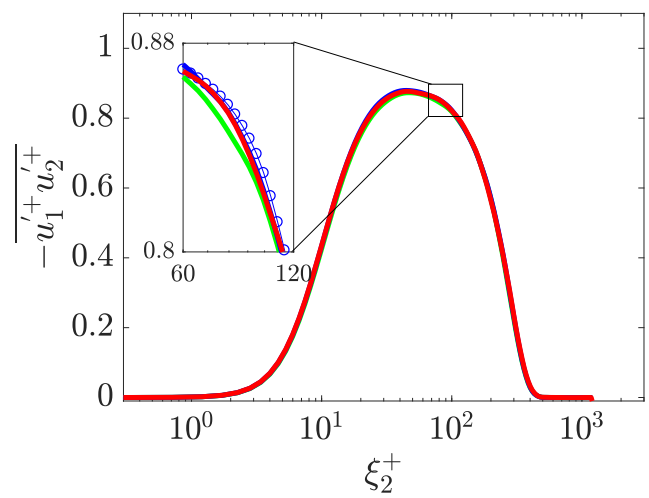

(d) Reynolds shear stress

FIG. 4. Inner scaled (a) mean streamwise velocity $\overline{u_{1}^{+}}$, (b) streamwise rms $\left(u_{1, \mathrm{rms}}^{+}\right)$, (c) wall-normal rms $\left(u_{2, \text { rms }}^{+}\right.$), and (d) Reynolds shear stress $-\overline{u_{1}^{\prime+} u_{2}^{\prime+}}$, averaged over time and spanwise direction $\left(\xi_{3}\right)$ for case BL_Simp at different streamwise locations and BL_Full at the middle of the domain. Symbols: (green) BL_Simp at $\xi_{1}=0$ (inlet); (blue) BL_Simp at $\xi_{1}=7.5 \delta_{99}$; (red) BL_Simp at $\xi_{1}=13 \delta_{99}$; (o) BL_Full at $\xi_{1}=7.5 \delta_{99}$.

of up to $10 \delta_{99}$ in the streamwise direction [32-34]. Lee and Sung (Ref. [15]) have found that these structures have a mean streamwise length of less than $6 \delta_{99}$ and that statistically, over $95 \%$ of the turbulent structures in their DNS had streamwise lengths of $<6 \delta_{99}$. And so, we opt for a domain of $40 \delta^{*} \sim 7 \delta_{99}$ in streamwise length.

The resolution is chosen so that the smallest turbulent structures can be adequately resolved. The streamwise and spanwise grids are uniform with $\Delta x^{+}=9, \Delta z^{+}=6$, which is comparable to the resolution parameters of Sillero et al. [16] $\left(\Delta x^{+} \approx 7, \Delta z^{+} \approx 4.7\right)$ and Orlu and Schlatter [22] $\left(\Delta x^{+} \approx 8.5, \Delta z^{+} \approx 4\right)$. The wall-normal domain uses a hyperbolic stretching with eight points in the viscous sublayer, $\left(\xi_{2}<5 \delta_{v}\right)$, with $\Delta y_{\min }^{+} \approx 0.3$. This is comparable to the wall-normal resolution of Sillero et al. [16] who also had eight points in the viscous sublayer at the inlet and that of Orlu and Schlatter [22] who had ten points in the viscous sublayer at their lowest Reynolds number. To improve accuracy, we opt to use fourth-order finite difference spatial operators. Appendix A compares the effect of both higher and lower finite difference spatial operators on case BL1460.

Cases BL2830, BL3550, and BL5650 are sampled over a period of $15 \delta_{99} / u_{\tau}$, whereas case BL1460 is sampled over a period of $30 \delta_{99} / u_{\tau}$. BL1460 was run for longer specifically to gather temporal statistics of the global quantities, e.g., skin friction coefficient and shape factor. 
TABLE III. DNS parameters for the turbulent boundary layer simulation cases.

\begin{tabular}{lcccc}
\hline \hline Dataset & $\mathrm{Re}_{\delta^{*}}$ & Initial condition & $\mathrm{Nx} \times \mathrm{Ny} \times \mathrm{Nz}$ & Sample time $\delta_{99} / u_{\tau}$ \\
\hline BL1460 & 1460 & Blasius + white noise & $300 \times 120 \times 160$ & 30 \\
BL2830 & 2830 & Fully turbulent field from BL1460 & $530 \times 160 \times 280$ & 15 \\
BL2830Wn & 2830 & Blasius + white noise & $530 \times 160 \times 280$ & 15 \\
BL2830Eig & 2830 & Blasius + white noise and streamwise mode & $530 \times 160 \times 280$ & 15 \\
BL2830H & 2830 & Fully turbulent field from BL5650 & $530 \times 160 \times 280$ & 15 \\
BL2830Sill & 2830 & Fully turbulent field from [16] & $530 \times 160 \times 280$ & 15 \\
BL3550 & 3550 & Fully turbulent field from BL2830 & $648 \times 230 \times 338$ & 15 \\
BL5650 & 5650 & Fully turbulent field from BL2830 & $968 \times 326 \times 512$ & 15 \\
\hline \hline
\end{tabular}

BL1460 uses a laminar boundary layer superimposed with white noise of fixed amplitude $0.1 u_{1, \infty}$ as an initial condition. Cases BL2830, BL3550, and BL5650 use fully turbulent fields from lower Reynolds number simulations as initial conditions. For example, BL5650 uses fields from BL2830, and BL2830 uses fields from BL1460. It will be shown in Sec. IV A that the statistically stationary solution is independent of the initial conditions used. The use of already turbulent fields as an initial condition greatly reduces the duration of the numerical transient when compared to using a laminar boundary layer as an initial condition.

To investigate the transient period, a set of turbulent cases is run at $\operatorname{Re}_{\delta^{*}}=2830$ with varying initial conditions. This specific Reynolds number was chosen to investigate the impact of using both higher and lower Reynolds number fields as initial conditions. The complete list of different cases is also shown in Table III. Each case has the same domain size and resolution as BL2830. BL2830Wn utilizes only white noise with an amplitude of $0.1 u_{1, \infty}$. Lower amplitudes of white noise are found to be insufficient to directly trigger turbulence and cause the flow to relaminarize, similar to what can occur in Lund-Wu-Squires (LWS) recycling [35,36]. BL2830Eig uses the same initial field as BL2830Wn but with a single superimposed streamwise mode as well as its corresponding wall-normal eigenfunction which are both based on stability analysis from Ref. [37]. Taking further inspiration from Ref. [17], cases BL2830 and BL2830H use fully turbulent fields from cases BL1460 and BL5650, respectively, after interpolating them to the appropriate resolution. Finally, case BL2830Sill uses an instantaneous data file from Ref. [16] at $\operatorname{Re}_{\delta^{*}}=5650$. A streamwise section of $\sim 7 \delta_{99}$ is taken and then interpolated to match the appropriate resolution.

\section{B. Implementation of $q_{0}^{\prime} / q_{0}$}

In practice, the ensemble averages used in Eq. (15) to calculate $q_{0}^{\prime} / q_{0}$ are approximated by spanwise and streamwise averages. From this value, the given metric terms are calculated, and the continuity and momentum equations [Eqs. (9) and (10)] are further solved at each time step. In this way, the simulation is fully independent of any a priori information and undercuts Ref. [17]'s original need for upstream stations. However, over the course of a simulation, computational errors may accumulate and cause $\delta^{*}$ to eventually drift. This could ultimately result in a substantial temporal drift in all integrated quantities and profiles. To prevent this, we implement a single relaxation term in the streamwise momentum equation to relax $\delta^{*}$ to a targeted value $\delta_{\mathrm{d}}^{*}$.

$$
\frac{\partial u_{1}}{\partial t}=-u_{j} \frac{\partial u_{1}}{\partial \xi_{j}}-\frac{1}{\rho} \frac{\partial P}{\partial \xi_{1}}+v \frac{\partial^{2} u_{1}}{\partial \xi_{j}^{2}}+\xi_{2} \frac{q_{0}^{\prime}}{q_{0}} u_{1} \frac{\partial u_{1}}{\partial \xi_{2}}+\frac{\alpha}{u_{1, \infty}} \frac{\left(\delta^{*}-\delta_{\mathrm{d}}^{*}\right)}{\delta^{*}} \xi_{2} u_{1} \frac{\partial u_{1}}{\partial \xi_{2}},
$$

where the timescale $\alpha=u_{\tau} / \delta_{99}$ is chosen so as to not introduce an additional timescale in the flow. It should be noted that this relaxation term is the only method of prescribing a specific $\delta^{*}$ to the simulation. Volumetric integration of Eq. (21) directly shows that the relaxation term describes to first order an exponential decay of the instantaneous $\delta^{*}$ towards the desired value. 


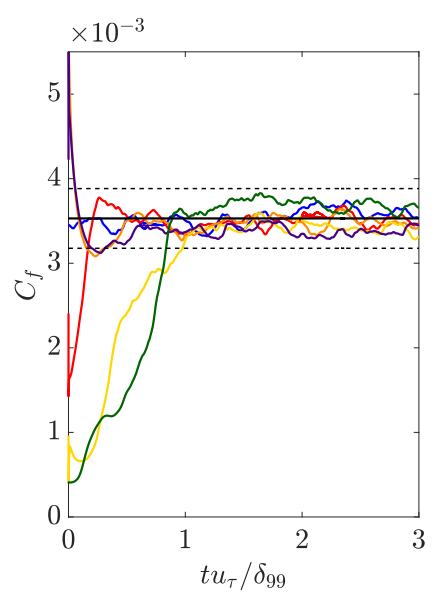

(a) Skin friction coefficient

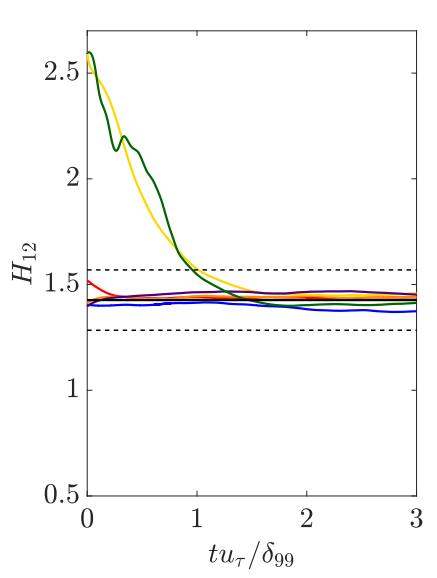

(b) Shape factor

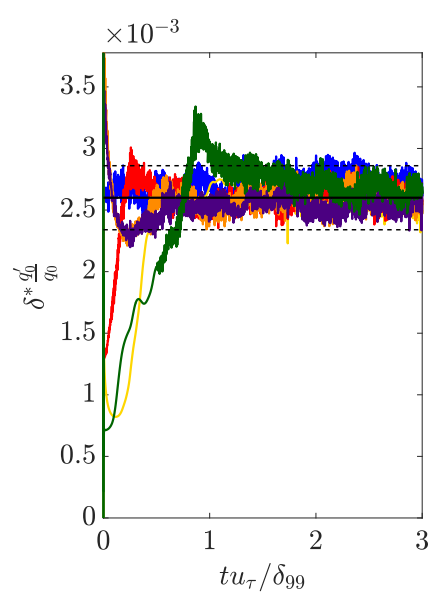

(c) Closure term

FIG. 5. Temporal evolution of (a) skin friction coefficient $C_{f}$, (b) shape factor $H_{12}$, and (c) normalized closure term $\delta^{*} \frac{q_{0}^{\prime}}{q_{0}}$, for the turbulent cases shown in Table III. Lines: (blue) BL2830 in the statistical steady state; (gold) BL2830Wn; (green) BL2830Eig; (red) BL2830; — (orange) BL2830H; (purple) BL2830Sill; (solid black) empirical values; (dashed black) $\pm 10 \%$ of empirical values for $C_{f}$ and $H_{12}$.

\section{RESULTS AND VERIFICATIONS}

Results from the simulation cases described in Table III are given in this section. In particular, integrated quantities such as shape factor $H_{12}$, skin friction coefficient $C_{f}$, and the various moments of $u_{1}$ are presented in comparison to values found in the literature.

\section{A. Transient behavior}

A major benefit of periodic boundary conditions is the independence of the statistical steady-state solution from the initial conditions. Here, we present transient behavior for turbulent boundary layers from a variety of initial conditions.

The shape factor is still calculated by Eq. (20) and the wall shear stress is still calculated by $\tau_{w}=$ $\left.\mu \overline{\partial u_{1} / \partial \xi_{2}}\right|_{\xi_{2}=0}$. However, for the current and following sections, the overbar will denote averaging in time and in both spanwise and streamwise directions. Figure 5 shows the transient behavior of each turbulent case listed in Table III. After a "wash-out" time, the flow reaches a statistical stationary state. It is clear that regardless of the initial condition, the integral quantities converge to the same statistical steady-state values. Moreover, cases BL2830Wn and BL2830Eig show a much slower convergence than the other cases by at least a factor of 4 . This provides justification for using lower Reynolds number turbulent fields as initial conditions for higher Reynolds number simulations, similar to extended Temporal DNS methods [38]. Case BL2830Sill demonstrates that even streamwise nonperiodic initial data can still be used as an initial condition to achieve the same results.

The transient period appears to last at most $2 \delta_{99} / u_{\tau}$ and can be as short as $0.5 \delta_{99} / u_{\tau}$. As expected, these transient periods are far shorter than with laminar initial fields. It has been argued by Nagib et al. [39] that the appropriate turbulent boundary layer timescale is $\delta_{99} / u_{\tau}$. Transient periods on the same order of magnitude are to be expected. To further quantify the temporal evolution, Figure 5(c) shows $\delta^{*} q_{0}^{\prime} / q_{0}$ over time for the cases presented in Table III. During the transient period, $\delta^{*} q_{0}^{\prime} / q_{0}$ appears to grow from its initial value to the nominal turbulent value.

Under statistically stationary conditions, the $C_{f}$ has an rms of $2.2 \%$ of its mean value and its fluctuations have an integral timescale of $\sim 0.3 \delta_{99} / u_{\tau}$ as shown in Fig. 6(a). In contrast, $H_{12}$ has 


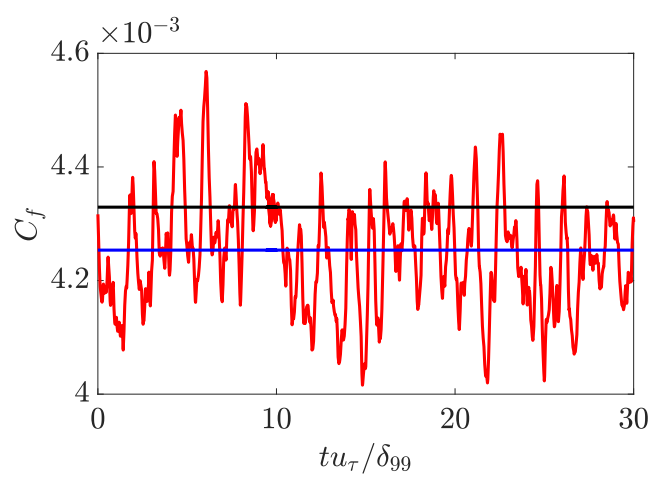

(a) Skin friction coefficient

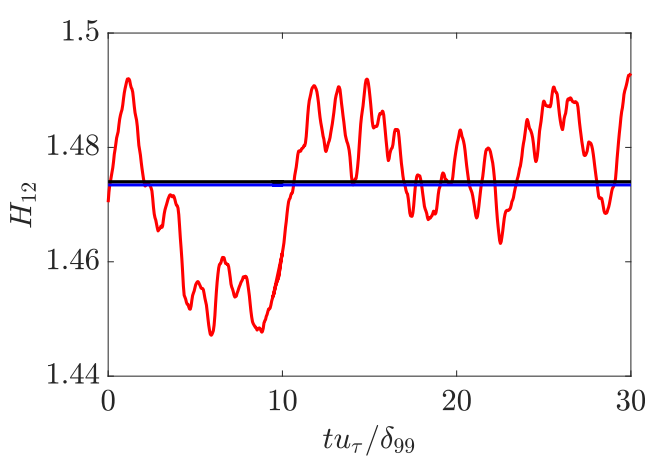

(b) Shape factor

FIG. 6. Temporal evolution of (a) $C_{f}$ and (b) $H_{12}$ for case BL1460. Colors: (red) instantaneous values, (blue) mean value, (black) empirical value.

an rms of $0.8 \%$ of its mean value with a much larger integral timescale of $\sim 1.9 \delta_{99} / u_{\tau}$, as shown in Fig. 6(b). Both of the integral timescales for skin friction and shape factor were calculated by first computing the two-time autocorrelation function during the statistically steady regime and then integrating the function only up to the autocorrelation function's first zero crossing. Naturally, $\delta^{*} q_{0}^{\prime} / q_{0}$ has fluctuations similar to $C_{f}$ rather than $H_{12}$ and these can already be seen in Fig. 5(c). These temporal oscillations are due to the approximation of ensemble averages by planar averages in the evaluation of Eq. (15).

\section{B. Integral and global quantities}

Monkewitz et al. [23] generated empirical fits of experiments for $H_{12}$ against Reynolds number and these will be used as a guide for appropriate values. Similarly, we use Chauhan's extended Coles-Fernholz skin friction relation

$$
\sqrt{\frac{2}{C_{f}}}=\frac{1}{\kappa} \ln \left(\mathrm{Re}_{\delta^{*}}\right)+C+D_{0} \frac{\ln \mathrm{Re}_{\delta^{*}}}{\operatorname{Re}_{\delta^{*}}}+\frac{D_{1}}{\mathrm{Re}_{\delta^{*}}}
$$

with $\kappa=0.384, C=3.3, D_{0}=182$, and $D_{1}=-2466$ [24]. While there is a broad variety of composite profiles, the previous empirical profiles use up-to-date experimental data and account for low Re effects.

The final values of $H_{12}$ obtained for the simulation cases are shown in Fig. 7(a) alongside the empirical fit in the numerical results with $1 \%$ error margins. Error bars were evaluated consistent with the method used in Ref. [40]. The agreement is very good and quite remarkable given the general scatter of experimental results [24]. Noticeably the calculated values are within $\pm 1 \%$ of the empirical values. Following suit, the skin friction coefficient, $C_{f}$, is compared against the extended Coles-Fernholz skin friction relation [24]. The results are shown in Fig. 7(b) with corresponding error bars, and the given simulations are within at most $\pm 3 \%$ of the empirical values. It is interesting to note that the present results are closer to the composite fit of the experimental data than the DNS results of Ref. [22].

\section{Mean velocity profiles}

Figure 8 displays the inner-scaled mean streamwise velocity profiles of the different Reynolds number simulation results plotted against those of [22]. Since the shape factors are in good agreement with empirical values, it is no surprise that the streamwise mean velocity profiles show 


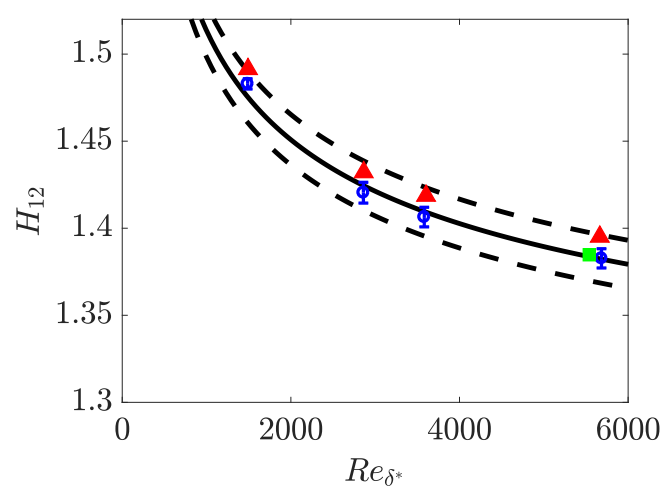

(a) Shape factor

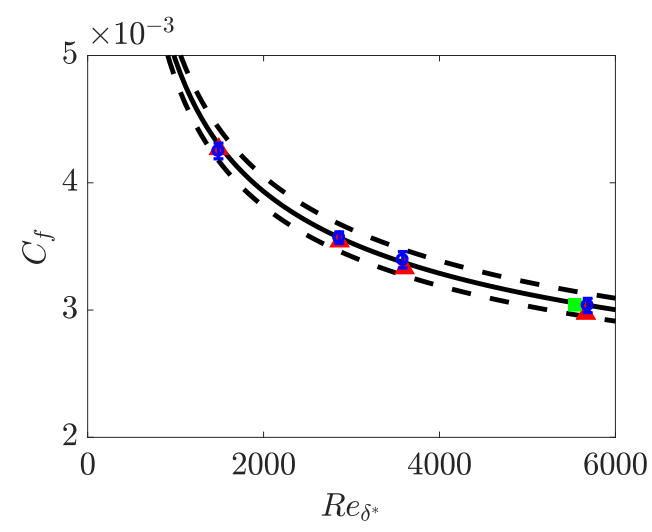

(b) Skin friction coefficient

FIG. 7. (a) Shape factor $H_{12}$ as a function of Reynolds number $\mathrm{Re}_{\delta^{*}}$. Solid line represents empirical fit by [23], and dashed lines indicate $\pm 1 \%$. (b) Skin friction as a function of $\operatorname{Re}_{\delta^{*}}$. Solid line represents the extended Coles-Fernholz relation with $\kappa=0.384, C=3.3, D_{0}=182$, and $D_{1}=-2466$ [24]. Dashed lines indicate $\pm 3 \%$. Symbols: $\triangle$ (red) DNS [22]; $\square$ (green) DNS [16]; $\circ$ (blue) current study.

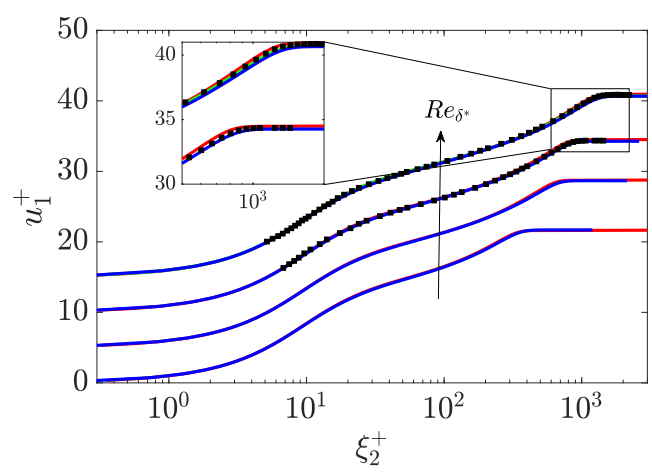

(a) streamwise velocity

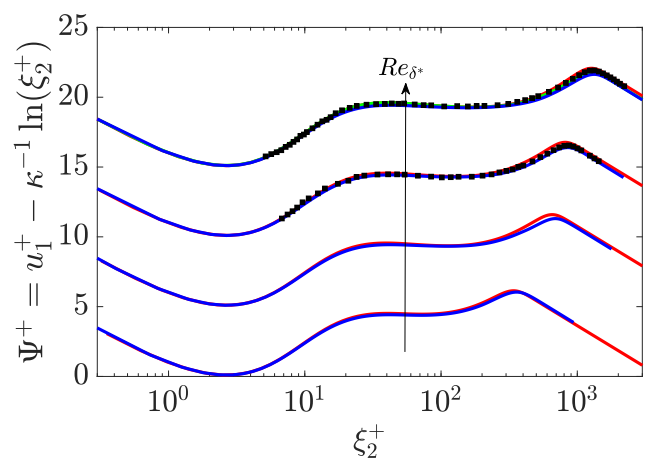

(b) Log-intercept function

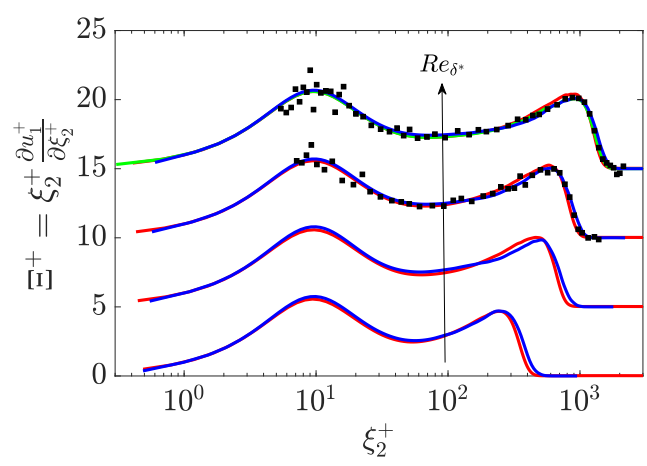

(c) Log-indicator function

FIG. 8. Mean inner scaled (a) streamwise velocity $\left(u_{1}^{+}\right)$, (b) log-intercept function $\Psi^{+} \equiv u_{1}^{+}-\kappa^{-1} \ln \left(\xi_{2}^{+}\right)$ with $\kappa=0.384$, and (c) $\log$-indicator function $\Xi \equiv \xi_{2}^{+} \frac{\partial u_{1}^{+}}{\partial \xi_{2}^{+}} \mathrm{vs} \xi_{2}^{+}$for different Reynolds numbers. From bottom to top: shifted by $5 u_{1}^{+}: \operatorname{Re}_{\delta}^{*}=1460,2830,3550,5650$. Legend: (red) [22] DNS data; (black) present work; (green) [16]; $\square$ (black) [22] experimental data. 


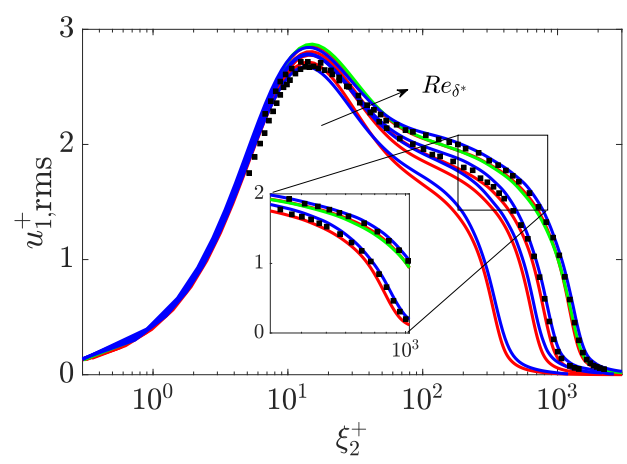

(a) Streamwise rms

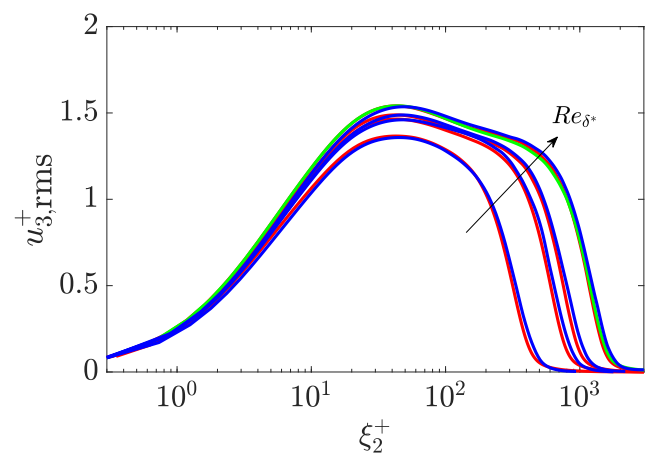

(c) Spanwise rms

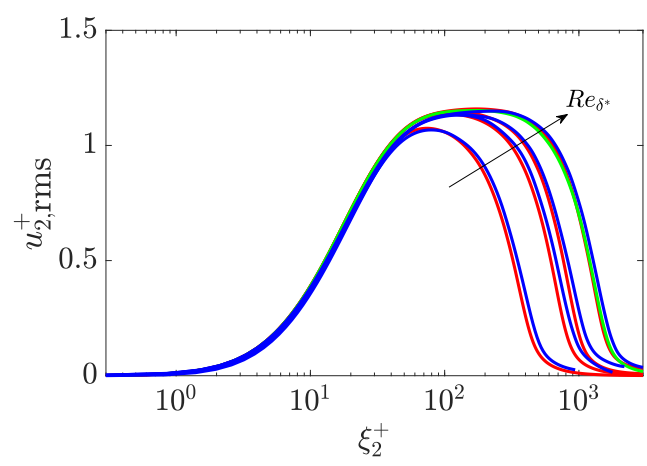

(b) Wall-normal rms

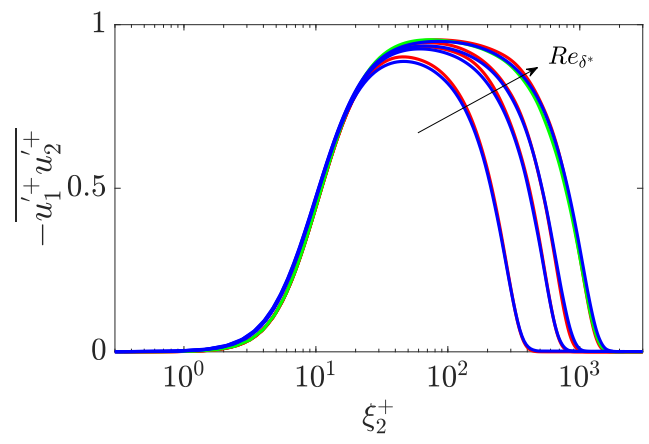

(d) Reynolds shear stress

FIG. 9. (a) $u_{1, \mathrm{rms}}^{+}$, (b) $u_{2, \mathrm{rms}}^{+}$, (c) $u_{3, \mathrm{rms}}^{+}$, and (d) $-\overline{u_{1}^{\prime+} u_{2}^{\prime+}}$ vs $\xi_{2}^{+}$for different Reynolds numbers. From bottom to top: $\operatorname{Re}_{\delta}^{*}=1460,2830,3550,5650$. Legend: (red) [22] DNS data; (blue) current study; (green) [16]; $\square$ (black) [22] experimental data.

similar agreement. The agreement of $u_{1, \infty}^{+}=\left(\frac{1}{2} C_{f}\right)^{-1 / 2}$ in the mean profiles makes it especially clear that the skin friction coefficients match.

Estimating the uncertainty for the mean profiles is more challenging than estimating the uncertainties for the skin friction coefficient $C_{f}$ and shape factor $H_{12}$. To reduce disk storage, we estimate the sampling error using the method provided by [41]. The maximum sampling error observed in $u_{1}^{+}$and $u_{1, \text { rms }}^{+}$for all cases was 0.003 and 0.01 , respectively.

We also compute the log-intercept function $\Psi^{+} \equiv u_{1}^{+}-\kappa^{-1} \ln \left(\xi_{2}^{+}\right)$and the log-indicator function $\Xi \equiv \xi_{2}^{+} d u_{1}^{+} / d \xi_{2}^{+}$. These two functions are often used to calculate the two constants required by the $\log$ law. A nominal value of $\kappa=0.384$ was used. Figure 8(c) shows that at lower Reynolds numbers, the log layer is not quite yet formed. This is indicative of inadequate scale separation. As the Reynolds number increases, however, a log layer appears to form around $\xi_{2}^{+}=40-100$ for $\operatorname{Re}_{\delta^{*}}=5650$. The log-intercept function is similarly constant in the log layer and also appears to plateau for $\xi_{2}^{+}=40-100$. Experimental data [22] are also plotted for both the log-intercept and log-indicator functions and show good agreement with the current simulations. In particular, near the wake region $\left(\xi_{2}^{+} \gtrsim 800\right)$, the profiles given for $\operatorname{Re}_{\delta^{*}}=3550$ agree better with experimental results than the corresponding profiles from the DNS of Ref. [22]. Reference [22] noted that due to low resolution near the wall, the velocity profile of the experimental data for $\xi_{2}^{+}<15$ is under-resolved. This is represented by a large scatter in experimental data for the log-indicator function.

The Reynolds stress and rms velocity profiles are shown with inner scaling in Figs. 9(a)-9(d). All of the profiles exhibit a near-wall peak, and the peak locations coincide with those of the profiles from Ref. [22]. There is a minor deviation in $u_{1, \mathrm{rms}}^{+}$in the log layer and wake region at around 
$\xi_{2}^{+} \approx 100-200$ of at most 5\%. The experimental results for $\operatorname{Re}_{\delta^{*}}=3550$ and 5650 [22] are also plotted and show good agreement with the current study in the region of deviation. Interestingly, in the region $\xi_{2}^{+}=100-200$, the experimental data for $\operatorname{Re}_{\delta^{*}}=3550$ stand at equal distance between our DNS results and the previous DNS results of Ref. [22]. The $u_{2, \mathrm{rms}}^{+}$profile features slightly higher values near the free stream. These fluctuations can be somewhat reduced by extending the domain in the wall-normal direction. However, in the present formulation, the $u_{2, \text { rms }}$ will never reach zero. Indeed, from integration of continuity [Eq. (9)], $u_{2, \infty}=\left(q_{0}^{\prime} / q_{0}\right) u_{1, \infty} \delta^{*}$. Since the closure for $q_{0}^{\prime} / q_{0}$ is not an a priori fixed quantity, $q_{0}^{\prime} / q_{0}$ varies in time, and hence the transpiration velocity also fluctuates in time. As mentioned previously, due to the low near-wall resolution of Ref. [22], the inner layer peak for experimental data at $\xi_{2}^{+} \sim 15$ is not fully captured. Finally, a small deviation may be observed in the spanwise and wall-normal rms velocity profiles in the near-wall region.

\section{COMPUTATIONAL COST}

The most popular method of boundary layer simulation remains the spatially growing boundary layer. However, this computational method is more computationally expensive than the current framework for three main reasons: an overall longer streamwise domain, slower statistical convergence, and smaller time step.

Spatially developing boundary layer simulations require large streamwise domains to reach the largest Reynolds numbers. Inflow generation methods, such as recycling and rescaling, heavily reduce the overall simulation cost by bypassing transition and increasing the initial Reynolds number. The recycling domain covers a large portion of the streamwise domain and for Ref. [16],

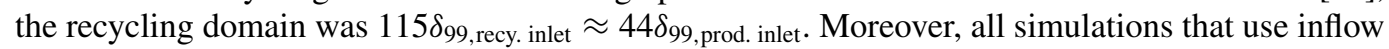
generation techniques must also undergo an "eddy-turnover recovery length" that increases with inlet Reynolds number [16]. Over this distance, none of the calculated statistics match empirical values within appropriate tolerance. For Ref. [16], this length scale encompassed over a quarter of their production domain $\left(27 \delta_{99}\right.$,prod. inlet out of a domain of $110 \delta_{99}$,prod. inlet $)$. In summary, a recycling and rescaling setup like that of Ref. [6] would therefore require an upstream domain length of at least $71 \delta_{99 \text {,prod.inlet }}$ or $48 \delta_{99,0}$, where $\delta_{99,0}$ is the boundary layer thickness at the location where statistics are extracted. Reference [22] required $56 \delta_{99,0}$ to reach the given Reynolds number as well. In contrast, our simulation at a Reynolds number of 5650 (BL5650) only required a streamwise domain of $7 \delta_{99,0}$. Therefore, the proposed method reduces the streamwise domain by about an order of magnitude.

Statistical convergence is another limiting factor of boundary layer simulation. Due to the growth of the boundary layer, the streamwise distance for which the boundary layer thickness remains within $\pm 0.5 \%$ of a specific value is approximately $1 \delta_{99}$. Thus streamwise developing boundary layer simulations are only able to average over streamwise slabs of approximately $1 \delta_{99}$ in streamwise length. In contrast, the current method uses a streamwise domain of $7 \delta_{99}$ in length and due to statistical homogeneity in the streamwise direction, the current method can average over a larger sample. In other words, the current method is expected to be seven times faster at converging statistics.

Finally, for a streamwise developing boundary layer DNS, the wall-normal resolution is determined by the inner layer thickness at the inlet (i.e., the lowest turbulent Reynolds number in the flow) so that all inner layer dynamics are fully resolved. Unfortunately, since the inner layer grows with streamwise distance, by the end of the production domain, the flow is unnecessarily over-resolved in the viscous sublayer. For example, the inner layer grew by $12 \%$ and by $20 \%$ for Refs. [16] and [22], respectively. In addition, the convective Courant-Friedrichs-Lewy number in the wall-normal direction is usually the leading constraint on the time-step size for flat-plate boundary layer DNS owing in large part to the wall-normal stretching of the mesh. Thus, Refs. [16,22] have time steps that could potentially be $20 \%$ and $12 \%$ larger, respectively, if the high fidelity simulations could be run on grids with the ideal resolution. Because the proposed method uses a $q^{\prime} / q$ calculated specifically to keep $\delta^{*}$ constant, it will maintain the initial boundary layer thickness. Since viscosity 
is prescribed and the free stream is far from the boundary layer, the method effectively specifies a single Reynolds number for the simulation. Therefore, an ideal resolution can be used and, consequently, a larger time step can be applied. Overall, the expected savings from a more ideal wall-normal resolution and larger time steps is at least $25 \%$.

The net cost reduction from the proposed method is about two orders of magnitude and is theoretically independent of the streamwise domain length of the proposed method. For example, although a domain $14 \delta_{99}$ in length would use twice the number of streamwise grid points, it would also have twice the sample range.

\section{CONCLUSION}

A method for simulating flat-plate turbulent boundary layers has been presented. The proposed method solves the Navier-Stokes equations in rescaled coordinates and was derived based on the assumption that self-similarity holds locally for a turbulent boundary layer. The method improves upon Spalart's original work by removing the dependence on lower Reynolds number simulations.

To derive the method, we rescaled the wall-normal coordinate by a single length scale, $q\left(x_{1}\right)$, and found by an a priori analysis that the additional terms resulting from rescaling counterbalances the Reynolds stresses in the wake region, thus keeping the boundary layer statistically stationary. To complete the derivation, we made two critical assumptions: (1) the modified Navier-Stokes equations hold over a narrow streamwise domain and (2) after rescaling, the flow is statistically stationary and statistically homogeneous in streamwise and spanwise directions. The method was then applied to a variety of Reynolds number cases, and the integral quantities and profiles were presented. The shape factor and skin friction coefficients were within $\pm 1 \%$ and $\pm 3 \%$ of aggregated experimental fits, respectively. Both the streamwise mean and rms velocity profiles showed good agreement with prior DNS and experimental values. Near $\xi_{2}^{+}=100$, the $u_{1, \mathrm{rms}}^{+}$compares favorably with the experimental values and deviates with respect to the DNS of [16] and [22] by about 5\%. The mean wall-normal velocity profile was also compared to that of Refs. [16,22]. Overall, the method was computationally less expensive than competing simulations by one to two orders of magnitude.

\section{ACKNOWLEDGMENTS}

The authors acknowledge the Texas Advanced Computing Center (TACC) at The University of Texas at Austin for providing computing resources that have contributed to the research results reported within this paper.

\section{APPENDIX A: ORDER OF ACCURACY}

Periodic flows are typically computed using spectral codes. The main results of this article use fourth-order finite difference operators. To verify that these spatial operators are adequate, case BL1460 was rerun with second- and sixth-order finite difference operators. The results are shown in Fig. 10.

The skin friction coefficients vary by less than $0.2 \%$. In terms of mean profiles, the deviation between all three methods was less than $1 \%$. This good agreement in mean profile carries over to the shape factors, which are within $0.5 \%$ of each other. The results with fourth- and sixth-order operators agree very well. There is a slight discrepancy in the mean profile at the overlap region $\xi_{2}^{+} \approx 500$ between the second-order and higher-order profiles.

\section{APPENDIX B: RELAMINARIZATION}

The current method can obtain a statistically stationary turbulent field from an initially laminar boundary layer superimposed with white noise. The converse is also possible: a laminar profile can be reobtained from a fully turbulent profile. A simulation was initialized with a turbulent field at $\operatorname{Re}_{\delta^{*}}=1460$ and then run with a high viscosity (corresponding to $\operatorname{Re}_{\delta^{*}}=100$ ). Instantaneous mean 


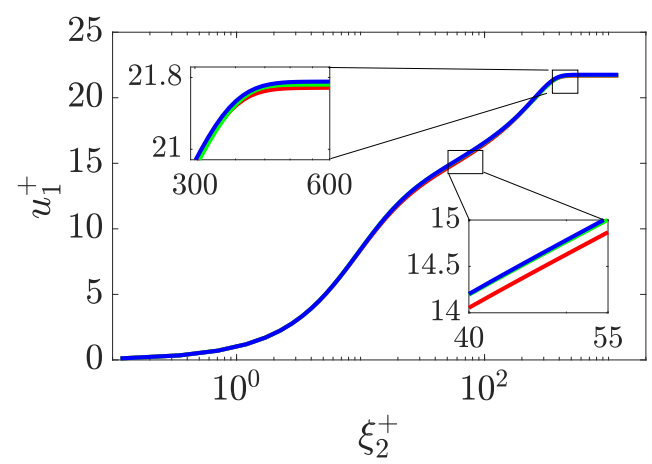

(a) stream-wise mean

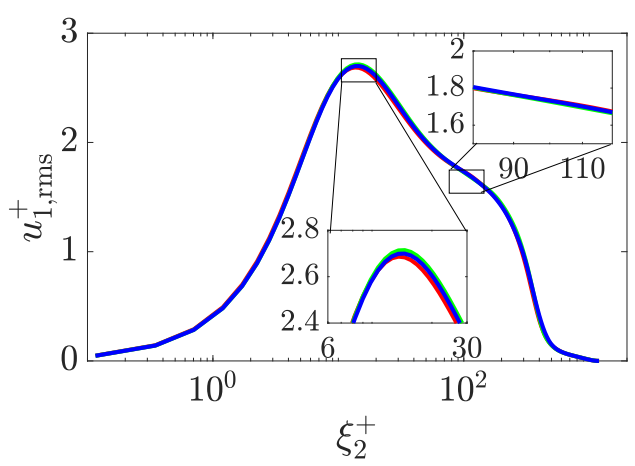

(b) stream-wise rms

FIG. 10. Streamwise mean (a) velocity and (b) rms profiles for $\mathrm{Re}_{\delta^{*}}=1460$ for different order spatial operators. Colors: (red) second order; (blue) fourth order; (green) sixth order.

velocity and rms profiles are computed through averaging in the streamwise and spanwise directions and are shown in Fig. 11.

The turbulent fluctuations quickly decay near the wall and more slowly far from the wall. The mean profile initially reduces its gradient near the wall and later steepens in the wake region. Eventually, the Blasius solution is obtained with zero residual turbulent fluctuations.

\section{APPENDIX C: TURBULENT KINETIC ENERGY}

The turbulent kinetic energy equation post evaluation at $x=x_{0}$ is given by

$$
\begin{aligned}
& \frac{\partial k}{\partial t}+\underbrace{\overline{u_{2}} \frac{\partial k}{\partial \xi_{2}}}_{\text {Turb. Advec. }}=-\underbrace{\frac{1}{\rho} \frac{\partial \overline{u_{2}^{\prime} p^{\prime}}}{\partial \xi_{2}}}_{\text {Pres. Diff. }}-\underbrace{\frac{1}{2} \frac{\partial \overline{u_{j}^{\prime} u_{j}^{\prime} u_{2}^{\prime}}}{\partial \xi_{2}}}_{\text {Turb. Diff. }} \\
& +\underbrace{v \frac{\partial^{2} k}{\partial \xi_{2}^{2}}}_{\text {Visc. Diff. }}-\underbrace{\overline{u_{i}^{\prime} u_{2}^{\prime}} \frac{\partial \overline{u_{i}}}{\partial \xi_{2}}}_{\text {Production }}-\underbrace{v \overline{\frac{\partial u_{i}^{\prime}}{\partial \xi_{j}} \frac{\partial u_{i}^{\prime}}{\partial \xi_{j}}}}_{\text {Dissipation }} \\
& + \text { Src Cont. }+H_{v} \text { Cont. }+H_{p} \text { Cont., }
\end{aligned}
$$

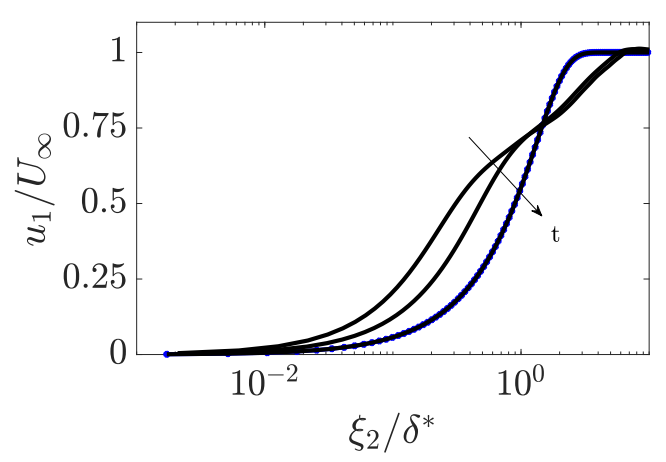

(a) Instantaneous mean

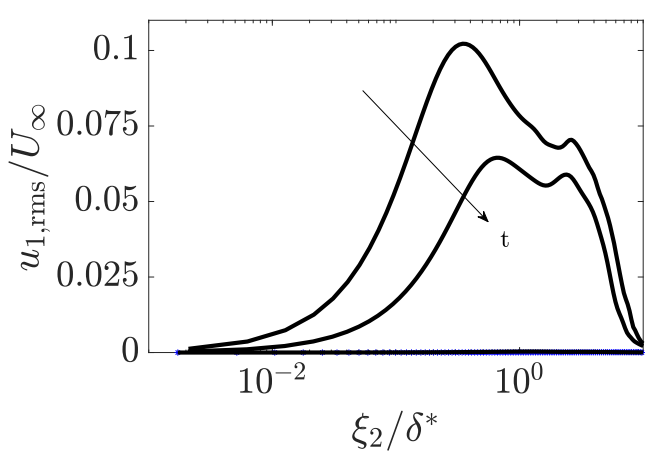

(b) Instantaneous rms

FIG. 11. Instantaneous (a) mean and (b) rms profiles for a laminarization of an initially turbulent boundary layer. Colors: ○ (blue) Blasius solution; (black) instantaneous profiles. 
where $k=1 / 2 \overline{u_{i}^{\prime} u_{i}^{\prime}}$ and the $\operatorname{Src}, H_{p}$ and $H_{v}$ contributions are given by

$$
\begin{aligned}
\text { Src Cont. }= & \frac{q^{\prime}}{q} \xi_{2}\left(\overline{u_{1}} \frac{\partial k}{\partial \xi_{2}}+\frac{1}{2} \frac{\partial \overline{u_{j}^{\prime} u_{j}^{\prime} u_{1}^{\prime}}}{\partial \xi_{2}}+\overline{u_{i}^{\prime} u_{1}^{\prime}} \frac{\partial \overline{u_{i}}}{\partial \xi_{2}}\right), \\
& H_{\mathrm{p}} \text { Cont. }=\frac{q^{\prime}}{q} \xi_{2} \frac{\partial \overline{u_{1}^{\prime} p^{\prime}}}{\partial \xi_{2}}, \\
H_{v} \text { Cont. }= & v\left(\xi_{2} \frac{q^{\prime}}{q}\right)^{2} \frac{\partial^{2} k}{\partial \xi_{2}^{2}} \\
& +v\left[2\left(\frac{q^{\prime}}{q}\right)^{2}-\frac{q^{\prime \prime}}{q}\right] \xi_{2} \frac{\partial k}{\partial \xi_{2}}+ \\
& \left.-v\left(\xi_{2} \frac{q^{\prime}}{q}\right)^{2} \frac{\partial u_{i}^{\prime}}{\partial \xi_{2}^{\prime}} \frac{\partial \xi_{i}^{\prime}}{\partial \xi_{2}}\right)+v \xi_{2} \frac{q^{\prime}}{q}\left(\frac{\partial u_{i}^{\prime}}{\partial \xi_{1}} \frac{\partial u_{i}^{\prime}}{\partial \xi_{2}}\right) .
\end{aligned}
$$

[1] L. Prandtl, Über flüssigkeitsbewegung bei sehr kleiner reibung, in Verhandlungen des III. Internationalen Mathematiker Kongresses Heidelberg, 8-13 August 1904 (Tubner, Leipzig, 1904).

[2] W. Schoppa and F. Hussain, Coherent structure generation in near-wall turbulence, J. Fluid Mech. 453, 57 (2002).

[3] N. Hutchins and I. Marusic, Evidence of very long meandering features in the logarithmic region of turbulent boundary layers, J. Fluid Mech. 579, 1 (2007).

[4] M. Lee and R. D. Moser, Direct numerical simulation of turbulent channel flow up to $\operatorname{Re}_{\tau} \approx 5200$, J. Fluid Mech. 774, 395 (2015).

[5] M. P. Schultz and K. A. Flack, Reynolds-number scaling of turbulent channel flow, Phys. Fluids 25, 025104 (2013).

[6] S. Pirozzoli and M. Bernardini, Probing high-Reynolds-number effects in numerical boundary layers, Phys. Fluids 25, 021704 (2013).

[7] W. J. Baars, N. Hutchins, and I. Marusic, Reynolds number trend of hierarchies and scale interactions in turbulent boundary layers, Philos. Trans. R. Soc. A 375, 20160077 (2017).

[8] Y. Mizuno and J. Jiménez, Mean velocity and length-scales in the overlap region of wall-bounded turbulent flows, Phys. Fluids 23, 085112 (2011).

[9] P. Schlatter, R. Örlü, Q. Li, G. Brethouwer, J. H. M. Fransson, A. V. Johansson, P. H. Alfredsson, and D. S. Henningson, Turbulent boundary layers up to $\mathrm{Re}=2500$ studied through simulation and experiment, Phys. Fluids 21, 051702 (2009).

[10] M. C. Adler, D. R. Gonzalez, C. M. Stack, and D. V. Gaitonde, Synthetic generation of equilibrium boundary layer turbulence from modeled statistics, Comput. Fluids 165, 127 (2018).

[11] Y. M. Chung and H. J. Sung, Comparative study of inflow conditions for spatially evolving simulation, AIAA J. 35, 269 (1997).

[12] T. S. Lund, X. Wu, and K. D. Squires, Generation of turbulent inflow data for spatially-developing boundary layer simulations, J. Comput. Phys. 140, 233 (1998).

[13] S. D. Mayor, P. R. Spalart, and G. J. Tripoli, Application of a perturbation recycling method in the largeeddy simulation of a mesoscale convective internal boundary layer, J. Atmos. Sci. 59, 2385 (2002).

[14] S. Stolz and N. A. Adams, Large-eddy simulation of high-Reynolds-number supersonic boundary layers using the approximate deconvolution model and a rescaling and recycling technique, Phys. Fluids 15, 2398 (2003).

[15] J. H. Lee and H. J. Sung, Very-large-scale motions in a turbulent boundary layer, J. Fluid Mech. 673, 80 (2011).

[16] J. A. Sillero, J. Jiménez, and R. D. Moser, One-point statistics for turbulent wall-bounded flows at Reynolds numbers up to 2000, Phys. Fluids 25, 105102 (2013). 
[17] P. R. Spalart, Direct simulation of a turbulent boundary layer up to $\mathrm{Re}=1410$, J. Fluid Mech. 187, 61 (1988).

[18] M. P. Martin, Direct numerical simulation of hypersonic turbulent boundary layers. Part 1. Initialization and comparison with experiments, J. Fluid Mech. 570, 347 (2007).

[19] L. Duan, I. Beekman, and M. P. Martín, Direct numerical simulation of hypersonic turbulent boundary layers. Part 2. Effect of wall temperature, J. Fluid Mech. 655, 419 (2010).

[20] L. Duan, I. Beekman, and M. P. Martín, Direct numerical simulation of hypersonic turbulent boundary layers. Part 3. Effect of Mach number, J. Fluid Mech. 672, 245 (2011).

[21] M. Kozul, D. Chung, and J. P. Monty, Direct numerical simulation of the incompressible temporally developing turbulent boundary layer, J. Fluid Mech. 796, 437 (2016).

[22] R. Örlü and P. Schlatter, Comparison of experiments and simulations for zero pressure gradient turbulent boundary layers at moderate Reynolds numbers, Exp. Fluids 54, 1547 (2013).

[23] P. A. Monkewitz, K. A. Chauhan, and H. M. Nagib, Self-consistent high-Reynolds-number asymptotics for zero-pressure-gradient turbulent boundary layers, Phys. Fluids 19, 115101 (2007).

[24] K. A. Chauhan, P. A. Monkewitz, and H. M. Nagib, Criteria for assessing experiments in zero pressure gradient boundary layers, Fluid Dyn. Res. 41, 021404 (2009).

[25] P. Spalart, M. Strelets, and A. Travin, Direct numerical simulation of large-eddy-break-up devices in a boundary layer, Int. J. Heat Fluid Flow 27, 902 (2006).

[26] O. Desjardins, G. Blanquart, G. Balarac, and H. Pitsch, High order conservative finite difference scheme for variable density low Mach number turbulent flows, J. Comput. Phys. 227, 7125 (2008).

[27] C. S. Vila, R. Vinuesa, S. Discetti, A. Ianiro, P. Schlatter, and R. Örlü, On the identification of wellbehaved turbulent boundary layers, J. Fluid Mech. 822, 109 (2017).

[28] M. P. Simens, J. Jiménez, S. Hoyas, and Y. Mizuno, A high-resolution code for turbulent boundary layers, J. Comput. Phys. 228, 4218 (2009).

[29] C. Tomkins and R. Adrian, Spanwise structure and scale growth in turbulent boundary layers, J. Fluid Mech. 490, 37 (2003).

[30] V. Priymak and T. Miyazaki, Long-wave motions in turbulent shear flows, Phys. Fluids 6, 3454 (1994).

[31] K. C. Kim and R. J. Adrian, Very large-scale motion in the outer layer, Phys. Fluids 11, 417 (1999).

[32] J. C. del Alamo, J. Jiminez, P. Zandonade, and R. D. Moser, Scaling of the energy spectra of turbulent channels, J. Fluid Mech. 500, 135 (2004).

[33] M. Guala, S. E. Hommena, and R. J. Adrian, Large-scale and very-large-scale motions in turbulent pipe flow, J. Fluid Mech. 554, 521 (2006).

[34] B. Balakumar and R. Adrian, Large- and very-large-scale motions in channel and boundary-layer flows, Philos. Trans. R. Soc. A 365, 665 (2007).

[35] A. Ferrante and S. Elghobashi, A robust method for generating inflow conditions for direct simulations of spatially-developing turbulent boundary layers, J. Comput. Phys. 198, 372 (2004).

[36] K. Liu and R. H. Pletcher, Inflow conditions for the large eddy simulation of turbulent boundary layers: A dynamic recycling procedure, J. Comput. Phys. 219, 1 (2006).

[37] L. M. Mack, Boundary-layer linear stability theory, Technical Report, Jet Propulsion Laboratory, 1984.

[38] T. Maeder, N. Adams, and L. Kleiser, Direct simulation of turbulent supersonic boundary layers by an extended temporal approach, J. Fluid Mech. 429, 187 (2001).

[39] H. M. Nagib, K. A. Chauhan, and P. A. Monkewitz, Approach to an asymptotic state for zero pressure gradient turbulent boundary layers, Philos. Trans. R. Soc. A 365, 755 (2007).

[40] K. J. Rah and G. Blanquart, Numerical forcing scheme to generate passive scalar mixing on the centerline of turbulent round jets in a triply periodic box, Phys. Rev. Fluids 4, 124504 (2019).

[41] T. A. Oliver, N. Malaya, R. Ulerich, and R. D. Moser, Estimating uncertainties in statistics computed from direct numerical simulation, Phys. Fluids 26, 035101 (2014). 